\title{
Measuring the Spatial and Temporal Diffusion of Urban House Prices---Taking the Case of 18 Cities in East China
}

\author{
He Wang1, a* and Chenyi Ma², b \\ 'Zijingang Campus of Zhejiang University, Hangzhou City, Zhejiang Province \\ ${ }^{2}$ Jiangqiao Town, Jiading District, Shanghai City \\ awanghelotus@163.com, bpartery@163.com
}

Keywords: House price; Spatial and temporal diffusion; Spatial VECM

\begin{abstract}
Based on spatial vector auto-regression model, this paper analyses the spatial and temporal diffusion of urban house prices in East China and examines the impact of price fluctuation of Shanghai and Hangzhou. The results show that assuming Shanghai as the price diffusion center city, more than half cities are affected significantly by the neighbor lag. Current price changes in Shanghai and Hangzhou have the biggest influence scope to other cities, while the house price in Hangzhou is the most difficult to be affected in the long-run. Compared with Shanghai, Hangzhou has greater and longer impact on other cities. It is concluded that the spatial and temporal diffusion exists in East China. Transportation accessibility, economic ties, information dissemination could explain house price diffusion in East China.
\end{abstract}

\section{Introduction}

With the acceleration of urbanization in China, the economic and social transactions between the big and medium cities have strengthened a lot, accompanied by the closer interactions between the real estate markets. East China, being as one of the fastest growing areas in China, its real estate market is always considered with great investment potential. Thus, whether there is a time and space diffusion in regional housing price fluctuation and how does the central city influences the housing price in surrounding cities could be interesting issues to be discussed.

The intrinsic relationships between regional housing prices have been widely concerned by scholars. Based on the vector autoregressive model and vector error correction model, Stevenson (2004) [1], Oikarinen (2004) [2] find the evidence of price fluctuations in different cities in Ireland and different regions in Finland. According to Pollakowski (1997) [3] and Luo \& Liu (2007)'s [4] research on real estate market in the United States and Australia, the neighboring cities will be more prone to generate housing price diffusion. Besides, Jones \& Leishman (2006) [5] point out that in the UK, the spill-over in housing price is more likely to happen in cities which have large population migration.

Based on the common VAR model and introduced with spatial relations, the spatial VAR model has been applied a lot to the study of house price diffusion effect. Ioannides \& Thanapisitikul researched house price change rate under six different geographical distance weights and found that the geographical distance has a powerful explanation to the change rate of house price, Cohen \& Ioannides(2016) [6]introduced transfer flow weight matrix on basis of that and proposed that the migration flow can explain the temporal and spatial diffusion of house prices to some extent. Holly \& Pesaran(2011)[7] confirmed the existence of strong time and spatial spillover effect in the UK's housing prices using Spatial VECM model and generalized impulse response function. It should be noted that the spatial matrix could be built based on many other factors except geographical connection, such as economic and social ties

Literature review on the study of domestic housing prices shows a focus on the study of the influence factors of house price fluctuation and lead-lag relationship between cities, quantitative research on the temporal and spatial diffusion effect of house price is very few, let alone quantitative research based on long time series data at the urban level. Therefore, this article aims at 
studying the diffusion of urban housing prices in East China using spatial VECM model, and explore the possible causes.

\section{Time and Spatial VECM Model of Housing Price}

Taking the house price series as endogenous vector and build the VAR and VECM models, the co-integration relationship between urban housing prices can be obtained. Now assume that there is a house price diffusion center in the city group, it differs from other cities since the impact of the outside world can be transmitted to the urban population at the same time through it. Based on the impact from the center city, the first order linear error correction model of other cities' housing is set as:

$$
\begin{aligned}
\Delta p_{i t}= & \phi_{i s}\left(p_{i, t-1}-\bar{p}_{i, t-1}^{s}\right)+\phi_{i 0}\left(p_{i, t-1}-p_{0, t-1}\right)+a_{i} \\
& +a_{i 1} \Delta p_{i, t-1}+b_{i 1} \Delta \bar{p}_{i, t-1}^{s}+c_{i 1} \Delta p_{0 t}+\varepsilon_{i t}
\end{aligned}
$$

Where $\boldsymbol{P}_{\mathbf{0}, t}$ stands for the housing price in center city, $\overline{\boldsymbol{p}}_{i, t}^{s}$ stands for the Spatial weighted value, which is defined as $\bar{p}_{i, t}^{s}=\sum_{j=\mathbf{0}}^{N} \boldsymbol{s}_{i j} \boldsymbol{p}_{j t}$, and $\sum_{j=\mathbf{0}}^{N} \boldsymbol{s}_{i j}=\mathbf{1}$.

As for the center city, namely $\mathrm{i}=0$, the model turns into:

$$
\Delta p_{0 t}=\phi_{0 s}\left(p_{0, t-1}-\bar{p}_{0, t-1}^{s}\right)+a_{0}+a_{01} \Delta_{p_{0, t-1}}+b_{01} \Delta \bar{p}_{0, t-1}^{s}+\varepsilon_{0 t}
$$

Combine Eq.1 and Eq.2, we can get:

$$
\Delta P_{t}=a+H P_{t-1}+\left(A_{1}+G_{1}\right) \Delta P_{t-1}+C_{0} \Delta P_{t}+\varepsilon_{t}
$$

Where $p_{t}=\left(p_{0 t}, p_{1 t}, \cdots p_{n t}\right)^{\prime}, a=\left(a_{0}, a_{1}, \ldots, a_{n}\right)^{\prime}, \varepsilon_{t}=\left(\varepsilon_{0 t}, \varepsilon_{1 t}, \ldots, \varepsilon_{N t}\right)^{\prime}$;

$$
\begin{aligned}
& \boldsymbol{H}=\left[\begin{array}{ccccc}
\phi_{0 s} & \mathbf{0} & \ldots & \mathbf{0} & \mathbf{0} \\
-\phi_{10} & \phi_{1 s}+\phi_{10} & \ldots & 0 & 0 \\
\vdots & \vdots & \ddots & \vdots & \vdots \\
-\phi_{N-1,0} & 0 & \ldots & \phi_{N-1, s+\phi_{N-1,0}} \\
-\phi_{N 0} & 0 & \ldots & 0 & 0 \\
\phi_{N s}+\phi_{N 0}
\end{array}\right]-\left[\begin{array}{c}
\phi_{0} S_{0}^{\prime} \\
\phi_{1 S} S_{1}^{\prime} \\
\vdots \\
\phi_{0} S_{N-1}^{\prime} \\
\phi_{0 S} S_{N}^{\prime}
\end{array}\right] \\
& A_{1}=\left[\begin{array}{ccccc}
a_{01} & 0 & \ldots & 0 & 0 \\
0 & a_{11} & \ldots & 0 & 0 \\
\vdots & \vdots & \ddots & \vdots & \vdots \\
0 & 0 & \ldots & a_{N-1,1} & 0 \\
0 & 0 & \ldots & 0 & a_{N, 1}
\end{array}\right], \quad G_{1}=\left[\begin{array}{c}
b_{01} s_{0}^{\prime} \\
b_{11} s_{1}^{\prime} \\
\vdots \\
b_{N-1,1 s_{N-1}^{\prime}}^{\prime} \\
b_{N 1 s_{N}^{\prime}}^{\prime}
\end{array}\right] \quad C_{0}=\left[\begin{array}{cccc}
0 & 0 & \cdots & 0 \\
c_{10} & 0 & \cdots & 0 \\
\vdots & \vdots & \vdots & \vdots \\
c_{N-1,0} & 0 & \cdots & 0 \\
c_{N, 0} & 0 & \cdots & 0
\end{array}\right]
\end{aligned}
$$

Where $S_{i}^{\prime}=\left(s_{i 0}, s_{i 1}, \ldots, s_{i N}\right)$.The matrix $A_{1}$ stands for the city's own short-term fluctuations, $\boldsymbol{G}_{1}$ represents the impact of short-term fluctuationcs in neighboring cities, and $\boldsymbol{C}_{\boldsymbol{0}}$ represents the current impact of changes in center city's housing prices on other cities.

\section{Research Objects Selection and Model Results}

This paper selects the residential sales price from January 2008 to February 2015 in the 18 cities in East China, namely, Shanghai, Hangzhou, Huzhou, Jiaxing, Shaoxing, Ningbo, Jinhua, Taizhou, Suzhou, Wuxi, Changzhou, Yangzhou, Nanjing, Nanchang, Fuzhou, Xiamen, Ji'nan, and.

At present, the Yangtze River Delta region has been formed a metropolitan system where Shanghai is considered as the regional center and Hangzhou,Nanjing as the sub-center, therefore, 
this paper set Shanghai as the center city of housing diffusion. Take the housing price data in logarithmic form and build the VECM model, the Eq. 1 and Eq. 2 could be turned into:

$$
\begin{aligned}
\Delta p_{0 t}= & \phi_{0 s}\left(p_{0, t-1}-\bar{p}_{0, t-1}^{s}\right)+\sum_{l=1}^{k 0 a} a_{0 l} \Delta p_{0, t-1}+\sum_{l=1}^{k 0 b} b_{0 l} \Delta \bar{p}_{0, t-1}^{s}+\varepsilon_{0 t} \\
\Delta p_{i t}= & \phi_{i s}\left(p_{i, t-1}-\bar{p}_{i, t-1}^{s}\right)+\phi_{i 0}\left(p_{i, t-1}-p_{0, t-1}\right)+\sum_{l=1}^{k i a} a_{i l} \Delta p_{i, t-1} \\
& +\sum_{l=1}^{k i b} b_{i l} \Delta \bar{p}_{i, t-1}^{s}+\sum_{l=1}^{k i c} c_{i l} \Delta p_{0, t-1}+c_{i 0} \Delta p_{0, t}+\lambda_{i} \widehat{\varepsilon}_{0 t}+\varepsilon_{i t}
\end{aligned}
$$

Where $\mathrm{A}$ is the residual of the urban housing price model in formula(4).Conduct a Wu-Hausman test to determine the exogenesis of the current price changes in central city.

Table 1 shows the regression results of the spatial VECM model with the assumption that Shanghai is a central city of diffusion ${ }^{\mathbb{1}}$. It can be seen that the long-run equilibrium relationship between Shanghai and Hefei has a significant impact on the short-term fluctuations in house prices in Hefei. Shanghai's housing price changes have a significant impact just on Jiaxing, Taizhou and Nanjing, Shanghai's current price changes have a significant positive effect on Hangzhou, Huzhou, Jiaxing and other 11 cities. Combined with Wu-Hausman test results, Shanghai shows a significant and exogenous effects on Hangzhou, Jiaxing, Ningbo and other 9 cities. In general, the housing price in Hangzhou, Huzhou, Jiaxing, Ningbo, Taizhou, Wuxi, Yangzhou, Nanjing, Shanghai, Fuzhou, Xiamen, Hefei are significantly affected by Shanghai.

The coefficient $\phi_{i s}$ shows that the housing price in Shanghai, Huzhou, Jiaxing and other 10 cities are negatively affected by the Long-run equilibrium relationship between the neighbors. The results in the fourth column shows that the house price in Shanghai, Hangzhou, Shaoxing and other 10 cities

\begin{tabular}{|c|c|c|c|c|c|c|}
\hline city & $\phi_{i 0}$ & $\phi_{i s}$ & $\begin{array}{l}\text { Delay-term of } \\
\text { neighbor city }\end{array}$ & $\begin{array}{l}\text { delay-term } \\
\text { of Shanghai }\end{array}$ & $\begin{array}{l}\text { current-term } \\
\text { of Shanghai }\end{array}$ & $\begin{array}{c}\text { Wu-Hausman } \\
\text { test }\end{array}$ \\
\hline Shanghai & - & $\begin{array}{c}-0.17^{* * * *} \\
(-2.8)\end{array}$ & $\begin{array}{c}0.80^{* * * *} \\
(2.8)\end{array}$ & - & - & - \\
\hline Hangzhou & - & - - - & $\begin{array}{c}0.86^{* * * *} \\
(2.9)\end{array}$ & $\begin{array}{c}-0.399 \\
(-1.425)\end{array}$ & $\begin{array}{c}0.37^{* * * *} \\
(3.40)\end{array}$ & 0.51 \\
\hline Huzhou & - & $\begin{array}{c}-0.19^{* * * *} \\
(-3.8)\end{array}$ & $\begin{array}{c}-0.02 \\
(-0.09)\end{array}$ & - & $\begin{array}{c}0.23^{* * * *} \\
(3.61)\end{array}$ & $-2.06^{* * *}$ \\
\hline Jiaxing & - & $\begin{array}{c}-0.38^{* * * *} \\
(-4.8)\end{array}$ & $\begin{array}{l}-0.66^{* *} \\
(-2.63)\end{array}$ & $\begin{array}{l}1.52^{* * * *} \\
(5.88)\end{array}$ & $\begin{array}{c}0.54^{* * * *} \\
(4.96)\end{array}$ & -0.72 \\
\hline Shaoxing & - & - & $\begin{array}{c}0.93^{* * * 4} \\
(3.28)\end{array}$ & $\begin{array}{c}-0.07 \\
(-0.44)\end{array}$ & $\begin{array}{c}0.17 \\
(1.24)\end{array}$ & -0.86 \\
\hline Ningbo & - & - & $\begin{array}{l}0.96^{* * * * 1} \\
(3.74)\end{array}$ & - & $\begin{array}{l}0.24^{*} \\
(1.98)\end{array}$ & 1.14 \\
\hline Jinhua & - & $\begin{array}{l}-0.15^{* * * *} \\
(-3.22)\end{array}$ & $\begin{array}{c}0.66 \\
(1.41)\end{array}$ & - & - & -0.41 \\
\hline Taizhou & - & $\begin{array}{l}-0.23^{\text {**** }} \\
(-3.30)\end{array}$ & $\begin{array}{c}-0.21 \\
(-0.68)\end{array}$ & $\begin{array}{c}0.97^{* * * *} \\
(3.89)\end{array}$ & $\begin{array}{l}0.29^{* *} \\
(2.17)\end{array}$ & -1.60 \\
\hline Suzhou & - & $\begin{array}{l}-0.19^{\text {**** }} \\
(-2.92)\end{array}$ & $\begin{array}{l}0.80^{* * * *} \\
(2.96)\end{array}$ & $\begin{array}{c}-0.15 \\
(-1.33)\end{array}$ & $\begin{array}{c}0.16 \\
(1.51)\end{array}$ & $1.98^{*}$ \\
\hline
\end{tabular}
are positively affected by the previous house price of surrounding cities.

Table.1 Estimation results of Spatial VECM with Shanghai as a diffusion center city

\footnotetext{
(1) Using the Spatial distance weighting matrix, the regression results of the main coefficients are listed in the table, which is calculated by Gauss. This model has also adopting a economic distance weight matrix and it shows a similar results.
} 


\begin{tabular}{|c|c|c|c|c|c|c|}
\hline Wuxi & - & - & $\begin{array}{c}0.34 \\
(1.32)\end{array}$ & $\begin{array}{c}0.25 \\
(0.99)\end{array}$ & $\begin{array}{l}0.41^{\text {*** }} \\
(3.82)\end{array}$ & -0.07 \\
\hline Changzhou & - & $\begin{array}{c}-0.127^{\text {**** }} \\
(-2.80)\end{array}$ & $\begin{array}{l}0.30^{*} \\
(1.72)\end{array}$ & - & $\begin{array}{c}0.06 \\
(1.00)\end{array}$ & $-2.78^{* * * *}$ \\
\hline Yangzhou & - & $\begin{array}{l}-0.18^{* * *} \\
(-2.57)\end{array}$ & $\begin{array}{c}0.59^{* * * *} \\
(2.97)\end{array}$ & - & $\begin{array}{l}0.20 \\
(3.769)\end{array}$ & $-2.56^{* * *}$ \\
\hline Nanjing & - & - & $\begin{array}{l}0.45 \\
1.58\end{array}$ & $\begin{array}{l}1.26^{* * * *} \\
(5.05)\end{array}$ & $\begin{array}{l}0.37^{\text {*** }} \\
(3.18)\end{array}$ & 0.10 \\
\hline Nanchang & - & $\begin{array}{l}-0.14^{* * * *} \\
(-3.771)\end{array}$ & $\begin{array}{c}0.18 \\
(0.75) \\
\end{array}$ & $\begin{array}{c}-0.04 \\
(-0.58)\end{array}$ & $\begin{array}{c}0.09 \\
(1.23)\end{array}$ & -0.91 \\
\hline Fuzhou & - & $\begin{array}{l}-0.12^{* * * *} \\
(-2.80)\end{array}$ & $\begin{array}{l}0.36^{* * *} \\
(2.27)\end{array}$ & - & $\begin{array}{l}0.31^{* * *} \\
(4.42)\end{array}$ & 0.40 \\
\hline Xiamen & - & - & $\begin{array}{c}0.36 \\
(1.21)\end{array}$ & - & $\begin{array}{l}0.20^{* *} \\
(2.19)\end{array}$ & 1.10 \\
\hline Jinan & - & - & $\begin{array}{c}0.52^{* * *} \\
(4.76)\end{array}$ & - & $\begin{array}{c}-0.02 \\
(-0.55)\end{array}$ & -0.46 \\
\hline Hefei & $\begin{array}{l}-0.12^{* * *} \\
(-5.08)\end{array}$ & - & $\begin{array}{c}0.22^{* * *} \\
(2.75)\end{array}$ & - & $\begin{array}{c}0.08^{\text {*** }} \\
(2.76)\end{array}$ & -0.52 \\
\hline
\end{tabular}

Note: $* * *$ indicates that the results are Significant at $1 \%$ level, $* *$ at $1 \%$ level and $*$ at $1 \%$ level. The maximum lag order is four; figures in brackets are t-statistics.

\section{Conclusions}

By constructing the spatial VECM model of the house price diffusion center, this paper examines the spatial spillover of housing price in East China, using residential sales price data of 18 cities from January 2008 to February 2015. The result shows that there is an obvious spatial and temporal diffusion effect of urban housing prices in East China. Shanghai, as the center of the house price diffusion, has a great impact on the house prices of neighboring cities. And compared with other cities, the impact from the Shanghai's current price range is the largest.

In East China, the population mainly flows to Shanghai, Hangzhou, Nanjing, Ningbo and other core cities, and this contradicts with the findings in foreign research, since the population there mainly move from high housing prices city to low housing prices. Therefore, the population migration shows no explanation for the diffusion effect of housing price. But the traffic accessibility between cities affects the housing price significantly. Since a close geographical distance and convenient transportation is always accompanied with a close linkage in the economy, society and culture, cities with adjacent relation show a closer relation in the housing price. Among them, the economic linkage is likely to be the main reason for the spillover of housing price. According to the economic linkage degree calculated from the economic data of 18 cities in 2014, Shanghai has the biggest radiation influence on other cities' economy, Thus, the strong economic ties between Shanghai and other cities may contribute to the housing price diffusion. In addition, the information dissemination and expectation may be another reason accounting for the housing spillover. The housing price information in big cities such as Shanghai, Hangzhou and Nanjing always attract great attention from neighboring regions, and the house price trend in these core city will influence the behavior in local real estate market, eventually cause the changes in housing price.

For the real estate industry practitioners and management departments, they should pay more attention to the interactive relationships between center city and its surroundings when observing the market. Under the current situation, it's necessary to take into consideration both the long-term differentiation trend and the short-term fluctuation when making policies in the real estate market. When there is an obvious house price fluctuation in center city, early warning should be taken. Moreover, the evolution law of real estate market and housing price response mechanism in center city should be watched closely to ensure the openness, transparency and accuracy of real estate 
information. In the long run, it's beneficial for the healthy development of real estate market to strengthen the accessibility and economic linkages between cities based on urban agglomeration or economic belt, optimize the allocation of market resources and establish a function complementary urban system.

\section{References}

[1] S. Stevenson. House price diffusion and inter-regional and cross - border house price dynamics [J]. Journal of Property Research, (2004), 21(4): 301-320.

[2] E. Oikarinen., The diffusion of housing price movements from center to surrounding areas[J]. Journal of Housing Research, (2004) 15(1): 3-28.

[3] H O. Pollakowski, T S. Ray,Housing price diffusion patterns at different aggregation levels: an examination of housing market efficiency[J]. Journal of Housing Research, (1997) 8: 107-124.

[4] Z Q Luo, C Liu, D. Picken, Housing price diffusion pattern of Australia's state capital cities [J]. International Journal of Strategic Property Management, (2007) 11(4): 227-242.

[5] C. Jones, C. Leishman, Spatial dynamics of the housing market: An interurban perspective [J]. Urban Studies, (2006), 43(7): 1041-1059.

[6] J. Cohen, Y M. Ioannides and W. Thanapisitikul. Spatial Effects and House Price Dynamics in the USA [J].Journal of Housing Economics. (2016): 1-13.

[7] S. Holly, M H. Pesaran and T Yamagata., The spatial and temporal diffusion of house prices in the UK [J]. Journal of Urban Economics, (2011) 69(1): 2-23. 